\title{
Focusing surface plasmons via changing the incident angle
}

Humeyra Caglayan, Irfan Bulu, and Ekmel Ozbay

Citation: Journal of Applied Physics 103, 053105 (2008); doi: 10.1063/1.2844552

View online: http://dx.doi.org/10.1063/1.2844552

View Table of Contents: http://aip.scitation.org/toc/jap/103/5

Published by the American Institute of Physics

\section{$A|P|$ Journal of Applied Physics}

Save your money for your research. It's now FREE to publish with us no page, color or publication charges apply.

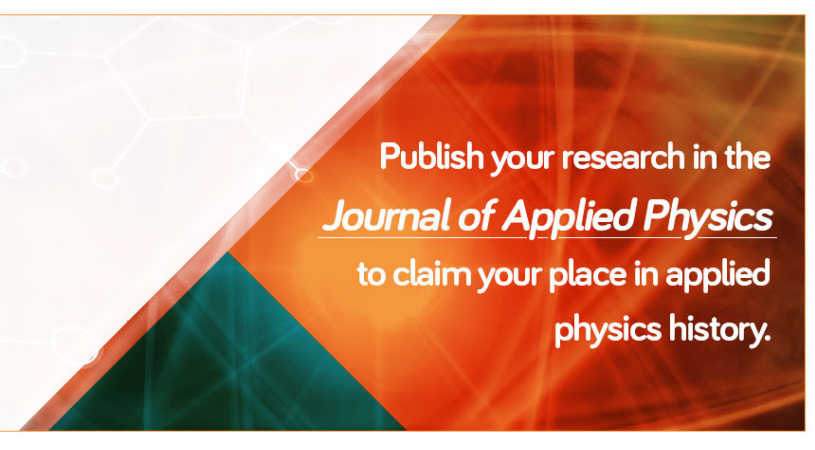




\title{
Focusing surface plasmons via changing the incident angle
}

\author{
Humeyra Caglayan, ${ }^{1, a)}$ Irfan Bulu, ${ }^{1,2}$ and Ekmel Ozbay ${ }^{1}$ \\ ${ }^{1}$ Nanotechnology Research Center-NANOTAM, Department of Physics and Department of Electrical \\ and Electronics Engineering, Bilkent University, 06800 Ankara, Turkey \\ ${ }^{2}$ School of Engineering and Applied Sciences, Harvard University, 29 Oxford Street, Cambridge, \\ Massachusetts 02138, USA
}

(Received 19 October 2007; accepted 14 December 2007; published online 7 March 2008)

\begin{abstract}
We report a circular metallic aperture with a subwavelength circular slit in the microwave regime, in which we experimentally demonstrate that this aperture can excite and focus surface plasmons. Under normal illumination, there is no focusing of the surface plasmons. However, by changing the incident angle, it is possible to focus surface plasmons. We showed that under a $20^{\circ}$ illumination angle surface plasmons focus at $4 \mathrm{~cm}$ away from the center on the surface of the aperture. (C) 2008 American Institute of Physics. [DOI: 10.1063/1.2844552]
\end{abstract}

\section{INTRODUCTION}

Surface plasmons (SPs) or surface waves are waves that propagate along the surface of a conductor. ${ }^{1}$ The electromagnetic field of the light interacts with the free electrons of the conductor surface and leads to the collective excitation of the electrons at the surface of a conductor in the longitudinal direction. This resonant collective surface charge oscillation constitutes the SPs. In the directions perpendicular to the interface, the SP intensity decays very fast in both neighboring media, whereas the SPs can propagate to large distances along the interface.

The increased interest in SPs stems from the recent advances that have enabled the manipulation of light on subwavelength scales and new possible applications such as highly integrated optical devices and circuits for high-speed communication technologies. Some of these elements have already been investigated such as plasmonic waveguides, mirrors, modulators, and switches based on the unique properties of SPs. ${ }^{2-4}$

In 1998, Ebbesen et al. experimentally demonstrated that an extraordinary transmission of light could be obtained through subwavelength hole arrays in metallic films. ${ }^{5}$ Their results have stimulated new research dedicated to the subject of enhanced transmission through periodic grating structures and enhanced transmission phenomena investigated from the optical to microwave regime. ${ }^{6-11}$ However, in all of these works, the role of SPs to achieve enhanced transmission effect was under investigation. On the other hand, there is not much work related to the focusing of SPs. Recently, Liu et al. showed that it is possible to focus surface plasmons and tune the focus of a plasmonic lens by changing the angle of the incident wave in the optical regime. ${ }^{12}$ Moreover, another group showed subwavelength focusing and the guiding of surface plasmons using a focusing array and nanowaveguide. ${ }^{13}$ However, to our knowledge, the focusing of SPs has not been investigated in the microwave regime. In this paper, we investigate a circular metallic aperture with a

${ }^{a)}$ Electronic mail: caglayan@fen.bilkent.edu.tr. subwavelength circular slit in the microwave regime and experimentally demonstrate that this aperture can excite and focus SPs.

\section{EXPERIMENT AND ANALYSIS}

In our experiments, we used a metallic (Al) aperture with a circular slit at $r=5 \mathrm{~cm}$. The width of the slit is $0.25 \mathrm{~cm}$ and the thickness of the sample is $1 \mathrm{~cm}$. The experimental setup consisted of an HP8510C network analyzer and a standard gain horn antenna to illuminate the electromagnetic waves between 8 and $12 \mathrm{GHz}$. We used a monopole antenna to measure the field on the surface of the aperture. The monopole antenna is obtained by removing $0.5 \mathrm{~cm}$ of the cladding from a coaxial cable and leaving the metal part.

Figure 1 shows the measured field on the surface of the aperture under normal illumination. The measured electric field intensity at $10.5 \mathrm{GHz}$ over a region of a $12 \times 12 \mathrm{~cm}^{2}$ area on the output side of the aperture is measured with a monopole antenna with a resolution of $0.1 \mathrm{~cm}$. Under normal illumination, the SPs guided through the center of the circular aperture but there is not focusing of SPs at a point. Figure 2(a) shows the simulation results of the metallic circular slit under normal illumination. We performed the theoretical simulations using commercial simulation software called CST MICROWAVE STUDIO. This program solves Maxwell equations in three dimensions with finite difference time domain (FDTD) method. As can be seen in Fig. 2(b), the experimental results are in good agreement with the FDTD calculations.

However, if the incident angle differs from the norm, one can obtain the focusing of SPs at a point. In order to investigate the focusing of SPs at a point via changing the incident angle, we inclined the incident radiation $20^{\circ}$ and measured the field amplitude on the surface of the aperture at $10.5 \mathrm{GHz}$. Figure 3 shows the measured field intensity under a $20^{\circ}$ illumination angle. The measured electric field intensity at $10.5 \mathrm{GHz}$ over a region of a $12 \times 12 \mathrm{~cm}^{2}$ area on the output side of the aperture is measured with a monopole antenna with a resolution of $0.1 \mathrm{~cm}$. The focusing of the surface waves can be clearly seen. The cross section along 

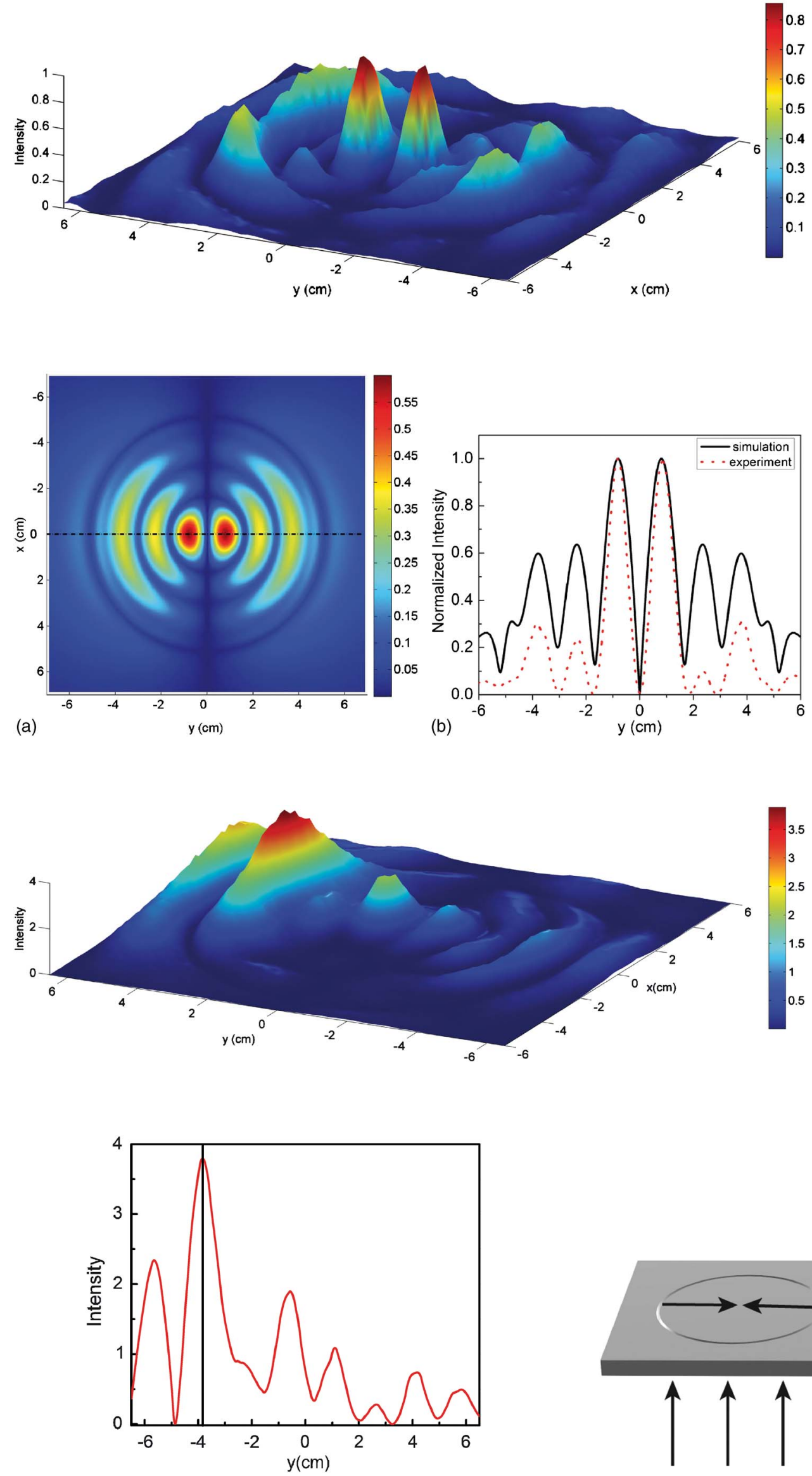

FIG. 4. (Color online) Measured field intensity along the focusing point under a $20^{\circ}$ inclined illumination. The focusing point is approximately $4 \mathrm{~cm}$ away from the center for a $20^{\circ}$ illumination angle.
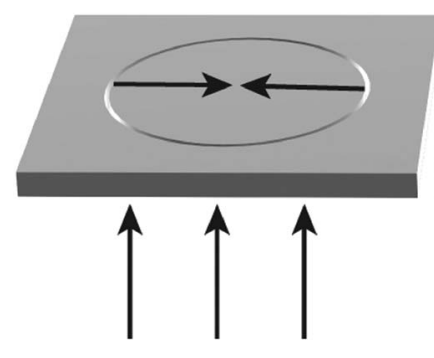

FIG. 1. (Color online) Measured electric field amplitude under normal illumination of metallic circular slit at $10.5 \mathrm{GHz}$. The measured electric field intensity over a region of a 12 $\times 12 \mathrm{~cm}^{2}$ area on the output side of the aperture is measured with a monopole antenna with a resolution of $0.1 \mathrm{~cm}$.

FIG. 2. (Color online) (a) Calculated electric field amplitude under normal illumination of metallic circular slit. (b) Comparison of the measured and calculated normalized intensity on the line shown in Fig. 2(a).

FIG. 3. (Color online) Measured electric field amplitude under a $20^{\circ}$ inclined illumination of metallic circular slit at $10.5 \mathrm{GHz}$. The measured electric field intensity over a region of a $12 \times 12 \mathrm{~cm}^{2}$ area on the output side of the aperture is measured with a monopole antenna with a resolution of $0.1 \mathrm{~cm}$.
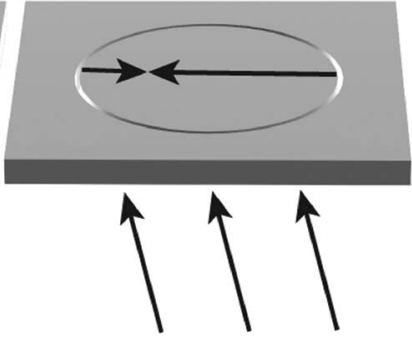

FIG. 5. Sketch of a metallic circular slit under normal and inclined illumination. Metallic (Al) apertures have a circular slit at $r=5 \mathrm{~cm}$. The width of the slit is $0.25 \mathrm{~cm}$ and the thickness of the sample is $1 \mathrm{~cm}$. 
the focusing point is illustrated in Fig. 4. The focusing point is approximately $4 \mathrm{~cm}$ away from the center for a $20^{\circ}$ illumination angle.

When electromagnetic waves are incident on a slit, most of the incoming waves are diffracted if the slit width is smaller than half of the wavelength of the incident waves. Because of this diffraction, the wave vector of these waves $\mathbf{k}$ change by $\Delta \mathbf{k}$ and in turn excite surface waves. These surface waves are guided through the normal of the slit. In the case of a circular slit, the waves are guided through the center of the circle in the normal illumination. When the incident angle differs from the norm, the change in the magnitude of the wave vectors influences the focusing of surface waves at a point (Fig. 5).

\section{CONCLUSION}

In conclusion, we presented the measured and calculated results of the field intensities on the surface of a metallic aperture under normal and inclined illumination. Our results show that under normal illumination there is no focusing of the SPs. However, by changing the incident angle, it is in fact possible to focus SPs. We showed that under a $20^{\circ}$ illumination angle, SPs focus at $4 \mathrm{~cm}$ away from the center on the surface of the aperture. The focusing of SPs can be used in many applications such as SP biosensors.
This work is supported by the European Union under the projects EU-NoE-METAMORPHOSE, EU-NoEPHOREMOST, and TUBITAK under Project Nos. 104E090, 105E066, 105A005, and 106A017. One of the authors (E.O.) also acknowledges partial support from the Turkish Academy of Sciences.

${ }^{1}$ H. Raether, Surface Plasmons on Smooth and Rough Surfaces and on Gratings (Springer-Verlag, Berlin, 1988).

${ }^{2}$ J. C. Weeber, Y. Lacroute, A. Dereux, E. Devaux, T. Ebbesen, C. Girard, M. U. Gonzalez, and A. I. Baudrion, Phys. Rev. B 70, 235406 (2004).

${ }^{3}$ H. Ditlbacher, J. R. Krenn, G. Schider, A. Leitner, and F. R. Aussenegg, Appl. Phys. Lett. 81, 1762 (2002).

${ }^{4}$ T. Nikolajsen, K. Leosson, and S. I. Bozhevolnyi, Appl. Phys. Lett. 85, 13 (2004).

${ }^{5}$ T. W. Ebbesen, H. J. Lezec, H. F. Ghaemi, T. Thio, and P. A. Wolf, Nature (London) 39, 667 (1998).

${ }^{6}$ H. J. Lezec, A. Degiron, E. Devaux, R. A. Linke, L. Martin-Moreno, F. J. Garcia-Vidal, and T. W. Ebbesen, Science 297, 820 (2002).

${ }^{7}$ H. Caglayan, I. Bulu, and E. Ozbay, Opt. Express 13, 1666 (2005).

${ }^{8}$ F. J. Garcia-Vidal, H. J. Lezec, T. W. Ebbesen, and L. Martin-Moreno, Phys. Rev. Lett. 90, 213901 (2003).

${ }^{9}$ S. S. Akarca-Biyikli, I. Bulu, and E. Ozbay, Appl. Phys. Lett. 85, 1098 (2004).

${ }^{10}$ A. P. Hibbins, J. R. Sambles, and C. R. Lawrence, Science 308, 670 (2005).

${ }^{11}$ H. Caglayan, I. Bulu, and E. Ozbay, J. Opt. Soc. Am. B 23, 419 (2006).

${ }^{12}$ Z. Liu, J. M. Steele, H. Lee, and X. Zhang, Appl. Phys. Lett. 88, 171108 (2006).

${ }^{13}$ L. Yin, V. K. Vlasko-Vlasov, J. Pearson, J. M. Hiller, J. Hua, U. Welp, D. E. Brown, and C. W. Kimball, Nano Lett. 5, 1399 (2005). 